\title{
AVISPA: a web tool for the prediction and analysis of alternative splicing
}

\author{
Yoseph Barash ${ }^{1,2,4,5^{*}}$, Jorge Vaquero-Garcia ${ }^{1,2}$, Juan González-Vallinas ${ }^{1,3+}$, Hui Yuan Xiong ${ }^{4 \dagger}$, Weijun Gao ${ }^{4}$, \\ Leo J Lee Lnd Brendan J Frey $^{4,5}$
}

\begin{abstract}
Transcriptome complexity and its relation to numerous diseases underpins the need to predict in silico splice variants and the regulatory elements that affect them. Building upon our recently described splicing code, we developed AVISPA, a Galaxy-based web tool for splicing prediction and analysis. Given an exon and its proximal sequence, the tool predicts whether the exon is alternatively spliced, displays tissue-dependent splicing patterns, and whether it has associated regulatory elements. We assess AVISPA's accuracy on an independent dataset of tissue-dependent exons, and illustrate how the tool can be applied to analyze a gene of interest. AVISPA is available at http://avispa.biociphers.org.
\end{abstract}

Alternative splicing (AS) is estimated to affect transcripts from over $95 \%$ of human multi-exon genes [1,2], with the most common class of AS involving cassette exons. Thousands of alternative cassette exons have been found to be differentially spliced between mammalian tissues, with tissues such as the brain displaying the most complex patterns $[1,2]$. These observations and the association of many splicing defects with diseases [3] motivated the recent derivation of a splicing code. The code, comprising a model with a set of rules that can predict splicing outcomes given genomic sequence and cellular context $[4,5]$, used over 1,000 regulatory features. Trained using inclusion measurements for 3,700 cassette exons across 27 mouse tissues, the code's model was shown to predict differential AS in four tissue groups: the central nervous system (CNS), muscle, digestive, and embryo versus adult tissues.

The derivation of a predictive splicing code served as proof-of-concept and enabled insights into RNA biogenesis $[5,6]$, but was limited in scope. Specifically, it was only applied to a subset of alternative exons in specific studies. However, given the importance of splicing in the

\footnotetext{
*Correspondence: yosephb@upenn.edu

${ }^{\dagger}$ Equal contributors

'Department of Genetics, University of Pennsylvania, Philadelphia, PA 19104, USA

${ }^{2}$ Department of Computer and Information Science, University of Pennsylvania, Philadelphia, PA 19104, USA

Full list of author information is available at the end of the article
}

study of gene regulation, development and disease, it became important to translate the splicing code models into a tool that would be accessible for researchers in a wide range of fields. Here, we present AVISPA (Advanced Visualization of Splicing Prediction and Analysis), a web tool that enables both prediction and splicing analysis of alternative and tissue-dependent exons in any gene of interest. Given an exon, the tool predicts whether it is alternative and whether its inclusion is expected to change in different tissues. It reports whether the exon is known to be alternative based on an internal transcripts database, and performs in silico splicing analysis, identifying putative regulatory elements and mapping those as tracks in the genome browser.

AVISPA's pipeline is illustrated in Figure 1. Users submit a query by specifying the sequence or genomic coordinates of either a single exon, or a triplet of exons that includes the immediate up- and downstream exons of the query exon. In the pre-processing step, the query is matched against an internal database of exon triplets mined from known transcripts and mapped to the reference genome. The result of the pre-processing is reported in the AVISPA's output and indicates existing evidence for whether the exon is alternatively spliced based on, for example, alignments of cDNA and EST data. After the query has been successfully matched, RNA features are extracted from the query exon and flanking regions [5]. At the first prediction stage, the 


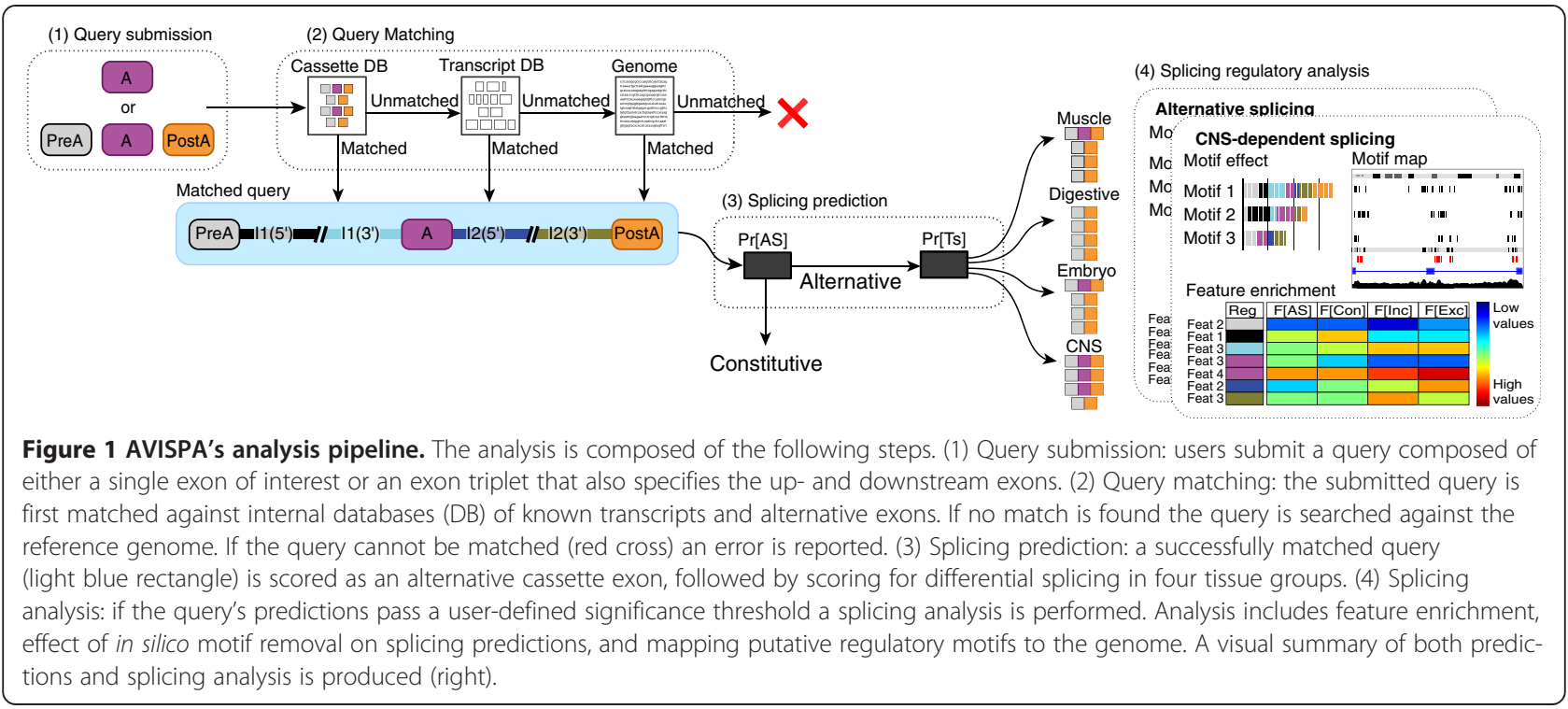

extracted features are used to predict whether the query exon is alternatively or constitutively spliced. If the query is predicted to be an alternative cassette exon, a second prediction step assesses whether the exon is differentially included in specific tissues.

The new web tool offers marked improvements over available software. First, it offers 'genome-wide' tissuedependent splicing predictions, where any exon can be submitted as a query. By contrast, the original work only allowed analysis on a previously mined set of approximately 12,000 cassette exons, while other tools focus on quantifying experimental data or general splice site and motif analysis [7-9]. Second, AVISPA offers a new in silico analysis of regulatory features and the mapping of putative regulatory sequence motifs in the genome. As part of this analysis, motifs found to be robustly included in the Bayesian ensemble of models and present in the query are removed in silico to determine their effect on splicing prediction. The relative effect of these feature removals is reported as a bar chart of the normalized feature effect (NFE). The putative regulatory motifs are also mapped to the genome using the UCSC genome browser, where they can be combined with other tracks, such as known single nucleotide polymorphisms and binding measurements of known splicing factors [10]. Additionally, the enrichment of the query's features is compared to reference groups such as alternative or constitutively spliced exons in AVISPA's database. Feature enrichment is reported using a standard heat map ranging from blue, for relatively low values, to red for relatively high values. For example, a relatively strong 3' splice site will appear red, indicating a high score, while a weak splice site will be marked blue.

The new tool also includes several other improvements. First, the prediction technique is now based on a
Bayesian neural network, which provides improved prediction accuracy compared to a battery of other methods [11]. Second, the original dataset of 3,700 cassette exons has been expanded to approximately 30,000 exons using data from 33 experiments in 11 mouse tissues [12]. Third, AVISPA uses an extended set of features that include computationally predicted nucleosome occupancy [13] together with primary sequence motifs implicated in general splicing regulation.

\section{Assessing splicing prediction accuracy}

The new two-stage prediction paradigm, combined with the expanded dataset, yields a significant improvement in detecting alternative cassette exons (Figure 2a). For example, using only tissue-dependent splicing predictors achieves an area under the curve (AUC) of $64 \%$ for distinguishing between alternative and constitutive exons, compared to $86 \%$ by the first stage classifier. The improved accuracy of $94 \%$ AUC achieved for detecting tissue-dependent exons is to be expected, as many regulatory features and higher intronic conservation are associated with such exons. Notably, AVISPA's sequence-based predictions offer a significant improvement compared to a similar classifier that directly uses normalized exon expression measurements from 33 experiments [12]. The latter achieves an overall lower accuracy of $71 \%$ AUC, with a significantly 2.5 -fold lower sensitivity (54\% versus $21 \%$ ) for high-confidence events at a false positive rate of $2 \%$. These results illustrate the usefulness of the new tool, which generalizes over experimental conditions and is not limited by technical factors such as microarray noise or read coverage. We note that these accuracy estimates can be considered as lower bounds, as some of the events labeled as constitutive in our database may be alternative. 


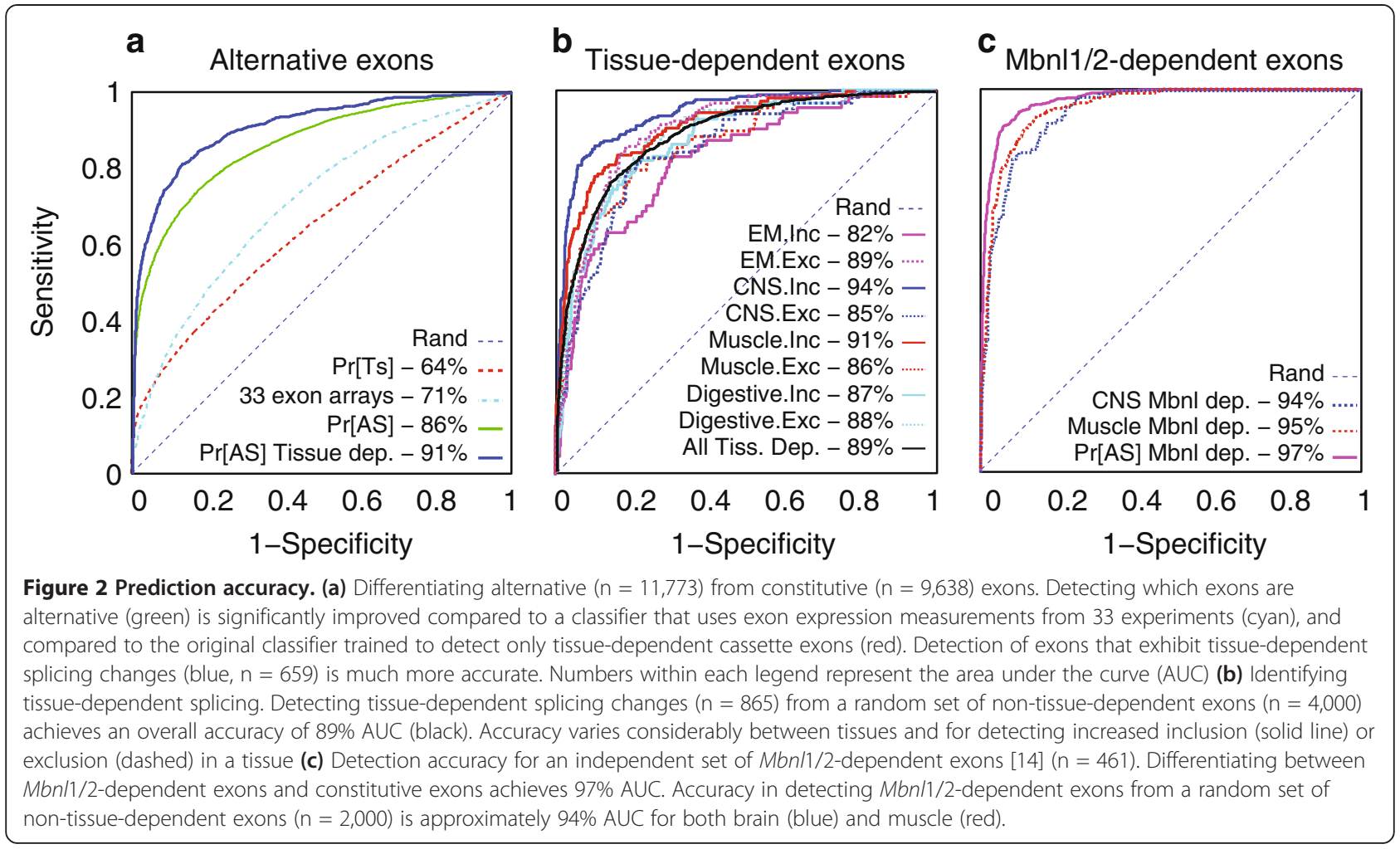

The new tool also achieves significant improvement in detecting tissue-dependent exons (Figure $2 b$ ). The overall accuracy in discriminating between tissue-dependent and non-tissue-dependent exons is $89 \%$ AUC, but varies considerably between tissues and between differential inclusion and exclusion in the same tissue type. For example, the highest accuracy was achieved for detecting increased inclusion of exons in CNS (94\% AUC) and muscle tissues (91\% AUC), while the lowest accuracy was for detecting increased exclusion in CNS (85\% AUC) and increased inclusion in embryonic tissues (82\% AUC).

In order to test AVISPA on an independent dataset, we computed predictions for a set of cassette exons recently shown to be regulated by the Muscleblind-like proteins Mbnl1/2 in mouse brain, muscle, and heart [14]. Figure 2c shows AVISPA easily distinguished these exons from constitutive exons (97\% AUC), similar to its performance in detecting tissue-dependent alternative exons in the original test set. In discriminating the Mbnl1/2-regulated exons from non-CNS- and nonmuscle-dependent exons, AVISPA achieves an AUC of $93 \%$ and $94 \%$, respectively, while in silico removal of $M b n l 1 / 2$ caused, on average, an almost two-fold larger effect for Mbnl1/2-regulated exons compared to the effect for non-muscle- and non-heart-dependent exons. The improved accuracy in detecting Mbnl1/2-regulated exons compared to the detection of tissue-dependent exons in the original test data is likely due to a lower false detection rate from the RNA-Seq and CLIP-Seq experiments in [14].

Finally, we also tested whether the regulatory features added in the web tool were useful for splicing prediction. As expected, many of the sequence motifs implicated in general splicing regulation were included in the code, especially for differentiating between alternative and constitutive exons. By contrast, the relation between nucleosome occupancy and alternative splicing is less well understood, and has garnered much research attention $[15,16]$. We found that the model selected features representing nucleosome occupancy around the alternative exon, but training the model without these features resulted in similar prediction accuracy (data not shown). This result indicates that other features in our model, such as di- and tri-nucleotide frequencies, already captured the 'predictive power' of computationally derived nucleosome position features.

\section{Vegfa in silico splicing analysis}

Previous work demonstrated how the splicing code model could be used to identify new regulatory elements, detect novel tissue-dependent splicing events, and study the evolution of splicing across vertebrates [6]. Here, we illustrate how the new tool can be used to analyze a well-studied gene of major interest. We applied AVISPA to the vascular endothelial growth factor A (Vegfa) gene. Vegfa has a complex and highly 
conserved pattern of alternative splicing that changes across tissues and developmental stages $[17,18]$. Its role in angiogenesis, which is controlled in part by alternative splicing, has made it an attractive target of several anticancer therapies. Accordingly, there is considerable interest in identifying the factors that regulate the splicing of Vegfa transcripts [18,19]. Analyzing all Vegfa exon triplets revealed that only exons 6 and 7 were predicted to be cassette exons, with a score corresponding to a false positive rate of 0.009 and 0.017 , respectively. For comparison, other exons scores corresponded to a false positive rate of 0.22 or higher (data not shown). These predictions are in line with annotated transcripts, many of which skip exon 6, one that skips exon 7 (ENSMUST00000113519), and several that skip both. Exons 6 and 7 were also both predicted, with a false positive rate of less than 0.025 , to exhibit differential splicing in all four major tissue groups modeled. While confidence in differential splicing was high, the predictions were not conclusive as to whether a relative increase or decrease of exon inclusion would occur in the tissues. These results reflect the conserved and complex splicing pattern of $V e g f a$, with RT-PCR experiments showing exon 6 to have a complex bi-phasic increase of inclusion in developing mouse and chicken heart [18]. Prediction of other splice variations of $V e g f a$, such as the 3 ' splice site variation in exon 8 , are currently not supported by the tool.

Figure 3 shows the regulatory feature analysis for differential inclusion of Vegfa exon 6 in muscle. The enrichment analysis in Figure $3 \mathrm{a}$ highlights that the alternative exon is depleted of non-tissue-specific exonic splicing enhancers and is highly enriched with exonic splicing silencers. Other highlighted features are enriched secondary structure-free regions in the upstream intron, a distant first AG nucleotide upstream and a particularly short preceding exon 5 . The preceding exon, for example, is $32 \mathrm{bp}$ long, and the enrichment analysis indicates that only $0.127 \%$ of the tool's reference set of alternative exons has a shorter preceding exon. The most dominant effect of in silico motif removal (Figure 3b) is for CU-rich elements known to bind Ptb1/

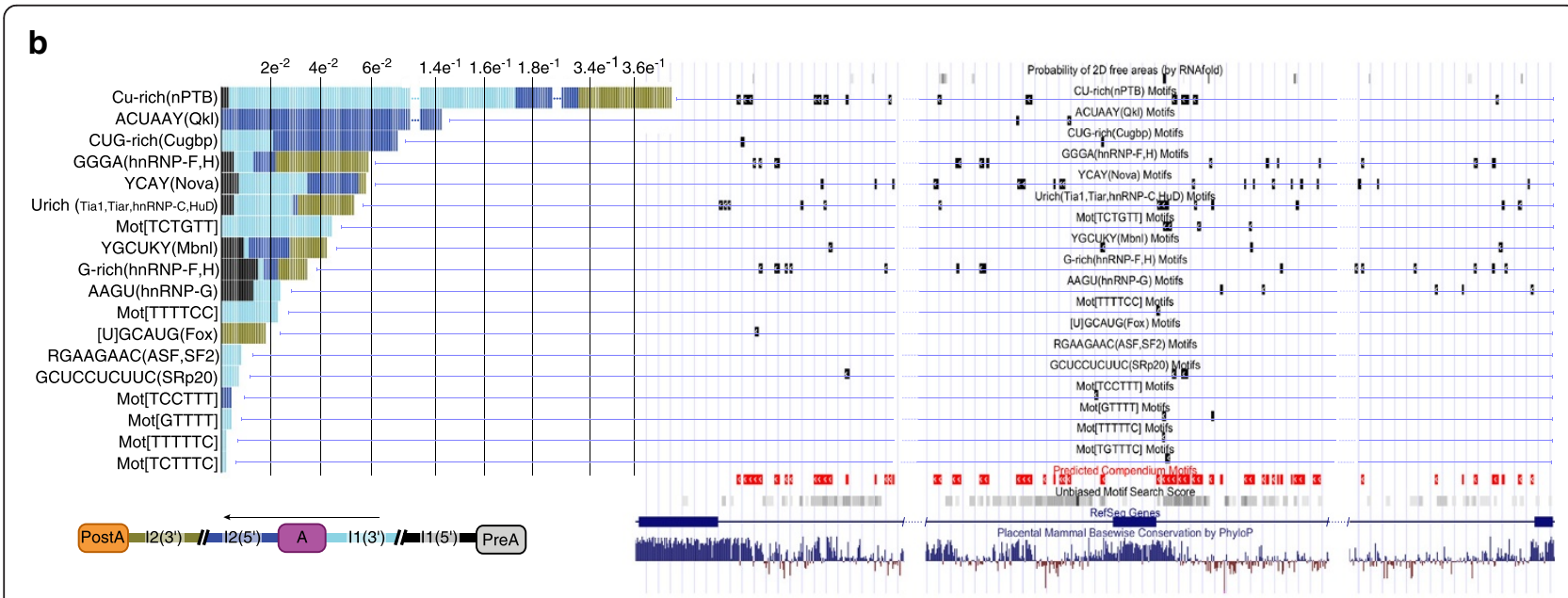

a

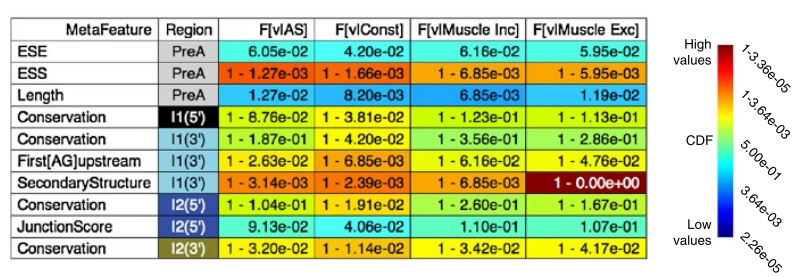

Figure 3 Analysis of Vegfa exon 6 muscle-dependent inclusion. A subset of the summary page produced by AVISPA is shown. (a) Feature enrichment analysis: the values of the features listed on the left are computed for Vegfa exon 6 and compared against matching feature values in a set of labeled exons. The four sets of exons compared against here are alternative exons ('AS', third column from the left), constitutive exons ('Const', third column from the right), exons differentially included in muscle ('Muscle Inc', second column from the right), and differentially excluded in muscle ('Muscle Exc', right most column). Relative enrichment or depletion of features is indicated using the heat map on the right. Only features with significantly low (blue) and high (red) values are shown here. The genomic region of each feature is indicated by the second from left column using the notation and colors in the top figure. (b) Stacked bar chart (left) of the normalized feature effect (NFE, y-axis) on splicing prediction. Only the top motifs are shown. Motif regions are annotated using the color scheme depicted below. Mapping of the motifs onto the UCSC genome browser is shown on the right. Tracks combining all motifs used by the code model (red), the unbiased motif search [5] (grey scaled), and conservation (blue) are added at the bottom. 
2, followed by an ACUAAY motif known to bind Quaking $(\mathrm{Qk})$. These splice factors have not been previously reported to regulate Vegfa, but a recent study estimates $39 \%$ of regulated exons during myogenesis are under the control of one or both of these splicing factors [20]. A smaller effect on splicing prediction in muscle is associated with intronic motifs known to bind Cugbp1/2 and Muscleblind-like protein (Mbnl1/2). Both Cugbp1/2 and Mbnl1/2 have been shown to play an important role in regulating splicing in developing hearts. Overexpressing Cugbp1 or knockdown of Mbnl1 in the adult mouse heart did not alter exon 6 inclusion levels significantly [18], but recent results point to possible compensatory effects between Mbnl1 and Mbnl2 [14]. Other elements implicated in Vegfa splicing regulation include the short YCAY motifs known to bind Nova proteins [21] and a UGCAUG motif, known to bind the brain- and musclespecific splicing factor Fox-1 (A2bp1) and its paralog Fox-2 (Rbm9) [22]. While the Fox-1/2 binding site is highly conserved, it resides over $1 \mathrm{~kb}$ downstream of exon 6 and $F o x-1 / 2$ have not been previously reported to regulate Vegfa. However, recent results indicate that Fox-2 knockdown in mice clearly alters Vegfa splicing pattern during heart development (Xiang-Dong Fu, personal communication). Smaller effects associated with non-tissue-specific regulation include G-rich elements, known to bind $h n R N P-F / H$, and U-rich elements that are known to bind hnRNP-C and Tiar/Tial [23]. Notably, Tia1 was previously reported to regulate Vegfa isoform expression [24]. Overall, our exploratory analysis of Vegfa splicing is consistent with previous results and offers new insights into mechanism of Vegfa regulation that are supported by recent experiments.

In summary, we presented a new tool, AVISPA, for in silico prediction and analysis of alternative splicing. The tool is not limited by technical constraints such as sequencing depth, and its predictions for alternatively spliced exons generalize over unmeasured conditions. Beyond the splicing outcome, it offers researchers the ability to identify putative regulatory elements and map those to the genome. These capabilities were recently used in an independent study to identify TIA1 as a regulator of an alternative exon coding miR-412 [25]. Here, we used a recent genome-wide study to demonstrate the tool's accuracy for predicting muscle, heart, and brain regulated exons and performed detailed in silico splicing analysis for the vascular endothelial growth factor A.

Several important elements remain as on-going and future enhancements of the tool. These include predictions for species other than mouse, predictions for additional forms of alternative splicing (for example, alternative 3' and 5' splice sites), and higher resolution of tissue specificity. Currently, AVISPA's predictions reflect confidence in alternative splicing or in relative, tissue- dependent, inclusion changes. Thus, users may infer an exon is likely to be alternative or to be differentially included in brain versus other tissues, but predictions for absolute inclusion levels (for example, $20 \%$ inclusion in brain, $40 \%$ inclusion in liver) are currently not supported. The tool has some technical limitations as well. Users can only submit a single cassette exon as a query, due to the computational burden involved in processing a query. Queries must be based on annotated exons, cannot contain exons shorter than 10 bases long, and non-canonical splicing by the minor spliceosome is not supported. Nonetheless, the ability to perform splicing prediction irrespective of experimental limitations, coupled with the new regulatory elements analysis, should serve researchers studying gene regulation, RNA biogenesis, and development. Moreover, AVISPA is built as a flexible platform that can be repeatedly updated as more data and improved models become available. The new computational analysis offered by AVISPA should facilitate the discovery of novel splicing variants, regulatory elements, and genomic variations affecting phenotypic variability or disease.

\section{Materials and methods}

\section{Query matching against sequence database}

The web-tool's internal database includes three components. The first is a database of 11,773 cassette exons that we previously mined from sequence libraries [5]. The second is a set of 9,638 exon triplets derived from Refseq [26] and other sequence libraries as described in [5], where every three constitutive exons in a transcript define a triplet. These triplets were also scanned against exon expression measurements in 11 mouse tissues [12] and triplets suspected to contain an alternative cassette exon were removed. A query's sequence is matched against the two transcript databases using BLAT with parameters set to tileSize $=8$, minMatch $=2$, minIdentity $=88$. The third database component is the mouse assembly $\mathrm{mm} 10$ from the UCSC Genome Browser [27]. Matching a query to the reference genome is executed only if no match in the two transcript-based databases is found, and only when genomic coordinates for all three exons are specified.

\section{Extended regulatory feature set}

We extended the set of putative regulatory features to include the occurrences of 350 new binding motifs in the seven regions around a cassette exon as defined in [5]. The motifs correspond to general splicing related RNA binding proteins (RBPs), SR and SR-related proteins (SC35, SRp20, 9G8, ASF/SF2, SRp30c, SRp38, SRp40, SRp55, SRp75, Tra2 $\alpha / \beta)$, and hnRNP proteins (hnRNPA1, hnRNPA2/B1, hnRNPF/H, hnRNPG).

We also added features encoding computationally predicted nucleosome occupancy around the alternative 
exon [13]. Features were defined as the average and maximal occupancy scores in the first 100 nucleotides in each intron and the first or last 50 nucleotides of the alternative exon.

\section{Extended training set for tissue-specific alternative splicing}

A total of 33 data tracks for normalized expression measurements using Affymetrix exon arrays were downloaded from the UCSC Genome Browser. The tracks are composed of measurements in 11 mouse tissues (brain, embryo, heart, kidney, liver, lung, muscle, ovary, spleen, testis, thymus) with three replicates for each tissue [12]. The expression of each exon and the relative inclusion of a putative cassette exon compared to its flanking exons were used as input features to train an ensemble of Bayesian neural networks [11]. The networks used these input features to identify differential inclusion and exclusion of alternative exons in the four tissue groups previously identified (CNS, muscle, digestive, embryo). Training was based on a subset of 3,770 cassette exons for which three probabilities for increased inclusion $\left(q^{\text {inc }}\right)$, increased exclusion $\left(q^{\text {exc }}\right)$ and no change $\left(q^{\text {nc }}\right)$ in each of the four tissue groups was previously computed [5]. This training step allowed the calibration of differential splicing estimation obtained from the new set of 33 experiments to the estimates used to train the original splicing model [5]. The model ensemble was then used to estimate differential splicing $\left(q^{\text {inc }}, q^{\text {exc }}, q^{\text {nc }}\right)$ for the remaining exons. The differential splicing estimates for the original set of 3,770 exons were averaged between the two datasets and care was taken to make sure predictions were based on non-overlapping training sets.

\section{Predicting alternative cassette exons using expression data and a single stage tissue-specific classifier}

The 33 expression data tracks described above were also used to train a Bayesian deep neural network classifier [11], denoted '33 exon arrays' in Figure 2a. Any exon triplets from the set of 11,773 cassette exons and 9,638 putative constitutive exons that had missing data were removed, maintaining a total of 8,986 for training and test purposes.

The prediction of alternative exons using a single stage tissue classifier, denoted $\operatorname{Pr}[T s]$ in Figure 2a, used a max function over the chance of differential splicing $\left(1-p^{\mathrm{nc}}\right)$ in each tissue.

Training a splicing code model for alternative exons and for tissue-dependent splicing

For the purpose of inferring a regulatory model, we used a Bayesian neural network that worked better for this task than support vector machines, boosted decision trees, and other leading machine learning techniques [11]. To discriminate between alternative and constitutive exons the network was set to have 10 hidden units and a sparsity prior of 0.9 for connections between features and hidden units. For predicting tissue-dependent splicing the network was set to have 20 units and a sparsity prior of 0.95 . Varying the sparsity prior between 0.85 and 0.95 and adding up to 10 more hidden units did not have a significant effect on the results (data not shown). An ensemble of 5,000 models generated by Markov chain Monte Carlo simulations was used to estimate differential splicing $\left(q^{\mathrm{inc}}, q^{\mathrm{exc}}, q^{\mathrm{nc}}\right)$ as was previously described [11].

\section{Scoring tissue-dependent splicing}

Under the new framework the probability that any given triplet of exons contain a tissue-dependent cassette exon can be expressed as:

$$
P\left(O^{t}=c h \mid r_{e}\right)=P\left(A S \mid r_{e}\right) P\left(O^{t}=c h \mid r_{e}, A S\right),
$$

where $P\left(O^{t}=c h \mid r_{e}\right)$ denotes the probability to observe a change in the exon's inclusion level in tissue $t$ given the exon's feature vector $r_{e}, P\left(A S \mid r_{e}\right)$ is the probability the exon is alternative, and $P\left(O^{t}=c h \mid r_{e}, A S\right)$ is the probability of observing differential splicing given that the exon is alternative. The first term on the right is computed by the first stage predictor, while the second term is computed by the second stage predictor.

\section{ROC performance evaluation}

Receiver operating characteristic (ROC) performance was evaluated using repeated five-fold cross-validation and care was taken to make sure predictions were based on non-redundant training sets, as was previously described [5]. Evaluation of discriminating between alternative and constitutive exons was based on a set of 11,773 cassette exons and 9,638 putative constitutive exons derived from EST/cDNA sequences [5]. In order to assess the accuracy of detecting cassette exons that exhibit a tissue-dependent splicing pattern (for example, differential inclusion in muscle) we compared the scores of such exons to those of a random set of exon triplets that do not exhibit this splicing pattern. The random set was selected using the following procedure. First, we used the 33 genome-wide exon expression measurements described above to quantify the inclusion level of all exon triplets from all Refseq transcripts. Next, we discarded triplets with missing data and required the relative expression of the upstream and downstream exons to be no more than 1.5-fold apart in all experiments. In order to avoid probe sets with little signal, we required the up- and downstream exons to have a normalized absolute value of at least 0.1 in at least 15 experiments. Additionally, we required in at least three experiments of the tissue group of interest (for example, digestive) that the up- and downstream exons are not in the 
bottom 20 percentile. Finally, the relative expression of each middle exon compared to its flanking exons was used to estimate the chance it is differentially included in each tissue group [28]. Any triplet that had a $P$-value of 0.7 or higher was deemed non-tissue-dependent and a set of approximately 2,000 exons was then selected for each tissue as a non-tissue-dependent exon set. Exons were selected randomly from the respective genes and then randomly from the relative order within the gene. We then verified that these are not biased in terms of relative location within the gene or gene length compared to a random sample of triplets from the genome (data not shown).

While small variations in the parameters of the above process did not have a notable effect on the results, we did detect an apparent selection bias in this procedure. Specifically, using expression measurements to select exons based on high confidence in non-tissue-dependent splicing may favor constitutive exons. Notably, the 'true' labels of any given exon as alternative or constitutive is unavailable. However, since our prediction algorithm has proved accurate in distinguishing alternative from constitutive exons (Figure 2a), we applied it to the set of 2,000 nontissue-dependent exons selected for each tissue group. Compared to a random set of 1,000 exon triplets, these exons were biased towards constitutive exon scores (Additional file 1). To correct for this apparent bias we subsampled 1,000 exons for each tissue group so that their scores as alternative match those in the random set (Additional file 1, green and red lines). This corrected set of a total of 4,000 predictions was then used for subsequent analysis (Figure 2b,c). We note that without this correction the initial set of non-tissue-dependent exons results in improved performance compared to that shown in Figure 2.

\section{In silico feature removal and normalized feature effect}

In order to evaluate the relative effect of a putative regulatory sequence motif (for example, the occurrence of a [U]GCAUG motif, known to bind Fox1/2, upstream of the alternative exon), the feature is first set to zero. The splicing predictions with the mutated feature, denoted $\left(p_{\Delta f}^{\mathrm{inc}}, p_{\Delta f}^{\mathrm{exc}}\right)$, are then computed with the total effect on differential splicing defined as $F E_{f}=\left|p^{\text {inc }}-p_{\Delta}^{\text {inc }}\right|+\mid p^{\text {exc }}$ $-p_{\Delta}^{\text {exc }} \mid$. This definition aims to capture the effect of features that not only change the confidence in a splicing change $\left(p^{\mathrm{nc}}-, p_{\Delta f}^{\mathrm{nc}}\right)$, but also change the relative confidence in either differential inclusion or exclusion. Finally, the normalized feature effect (NFE) is defined as:

$$
N F E_{f}=\frac{F E_{f}}{\sum_{j \in J} F E_{j}}
$$

where $J$ is the set of robust features. By itself, the NFE has no statistical significance measure associated with it. The
NFE serves mainly as a quantitative tool to guide researchers interested in knowing which of the identified regulatory features have a higher effect on the model's prediction confidence.

\section{Additional file}

\begin{abstract}
Additional file 1: Figure S1. Correcting constitutive exons selection bias in non-tissue-dependent exons. Exon scores for being alternative versus constitutive ( $\mathrm{x}$-axis) are plotted as a cumulative distribution function (CDF, $y$-axis). The initial set of selected non-tissue-dependent exons (blue) was biased towards constitutive exons compared to a random sample of 1,000 exon triplets from the genome (red). Subsampling the original set of 2,000 exons per tissue to fit the score distribution of a random set gave a good fit (green). Both green and red line plots are accumulated over all exons in all tissues as no significant difference was observed between the different tissues.
\end{abstract}

\section{Abbreviations}

AS: Alternative splicing; AUC: Area under the curve; AVISPA: Advanced visualization of splicing prediction and analysis; CNS: Central nervous system; EST: Expressed sequence tag; NFE: Normalized feature effect.

\section{Competing interests}

The authors declare that they have no competing interests.

\section{Authors' contributions}

$Y B$ and BJF conceived of the project. YB developed the combined prediction framework and in silico feature analysis. YB, JVG and WG developed the analysis pipeline with input from all authors. YB, JVG, WG and LJL created the sequence databases. JGV, WG and JVG developed the web tool. HYX, YB and BJF developed the prediction algorithms. YB and JVG performed the data analysis. YB wrote the paper with input from BJF. All authors read and approved the final manuscript.

\section{Acknowledgements}

We thank Ben Blencowe for his support and advice throughout this project. We thank Kristen Lynch for helpful feedback and discussions; Xiang-Dong Fu for sharing experimental results; members of the Barash, Lynch, Blencowe and Frey labs for providing helpful comments on the manuscript and suggestions for the web tool. Funding to JGV through FPI grant from the Spanish Ministry of Science; HYX, WG and LL were funded from NSERC Steacie and CIHR grants to BJF. While at the University of Toronto, YB was funded from an OGI Spark grant and a Genome Canada grant to BJF and others.

\section{Author details}

${ }^{1}$ Department of Genetics, University of Pennsylvania, Philadelphia, PA 19104, USA. ${ }^{2}$ Department of Computer and Information Science, University of Pennsylvania, Philadelphia, PA 19104, USA. ${ }^{3}$ Universitat Pompeu Fabra, Barcelona 08003, Spain. ${ }^{4}$ Department of Electrical and Computer Engineering, University of Toronto, Toronto, ON M5S 3G4, Canada. ${ }^{5}$ Banting and Best Department of Medical Research, University of Toronto, Toronto, ON M5G 1L6, Canada.

Received: 9 July 2013 Accepted: 11 October 2013 Published: 24 October 2013

\section{References}

1. Pan Q, Shai O, Lee LJ, Frey BJ, Blencowe BJ: Deep surveying of alternative splicing complexity in the human transcriptome by high-throughput sequencing. Nat Genet 2008, 40:1413-1415.

2. Wang ET, Sandberg R, Luo S, Khrebtukova I, Zhang L, Mayr C, Kingsmore SF, Schroth GP, Burge CB: Alternative isoform regulation in human tissue transcriptomes. Nature 2008, 456:470-476.

3. Wang ET, Cooper AT: Splicing in disease: disruption of the splicing code and the decoding machinery. Nature 2007, 8:749-761.

4. Wang Z, Burge CB: Splicing regulation: from a parts list of regulatory elements to an integrated splicing code. RNA 2008, 14:802-813. 
5. Barash Y, Calarco JA, Gao W, Pan Q, Wang X, Shai O, Blencowe BJ, Frey BJ: Deciphering the splicing code. Nature 2010, 465:53-59.

6. Barbosa-Morais NL, Irimia M, Pan Q, Xiong HY, Gueroussov S, Lee LJ, Slobodeniuc V, Kutter C, Watt S, Colak R, Kim T, Misquitta-Ali CM, Wilson MD, Kim PM, Odom DT, Frey BJ, Blencowe BJ: The evolutionary landscape of alternative splicing in vertebrate species. Science 2012, 338:1587-1593.

7. Yeo G, Burge CB: Maximum entropy modeling of short sequence motifs with applications to RNA splicing signals. J Comput Biol 2004, 11:377-394.

8. Dogan Rl, Getoor L, Wilbur WJ, Mount SM: SplicePort-an interactive splicesite analysis tool. Nucleic Acids Res 2007, 35:W285-W291.

9. Cartegni L, Wang J, Zhu Z, Zhang MQ, Krainer AR: ESEfinder: a web resource to identify exonic splicing enhancers. Nucleic Acids Res 2003, 31:3568-3571.

10. Yeo GW, Coufal NG, Liang TY, Peng GE, Fu X-D, Gage FH: An RNA code for the FOX2 splicing regulator revealed by mapping RNA-protein interactions in stem cells. Nat Struct Mol Biol 2009, 16:130-137.

11. Xiong HY, Barash Y, Frey BJ: Bayesian prediction of tissue-regulated splicing using RNA sequence and cellular context. Bioinformatics 2011, 27:2554-2562.

12. Pohl AA, Sugnet CW, Clark TA, Smith K, Fujita PA, Cline MS: Affy exon tissues: exon levels in normal tissues in human, mouse and rat. Bioinformatics 2009, 25:2442-2443.

13. Xi L, Fondufe-Mittendorf $Y$, Xia L, Flatow J, Widom J, Wang JP: Predicting nucleosome positioning using a duration Hidden Markov Model. BMC Bioinformatics 2010, 11:346.

14. Wang ET, Cody NAL, Jog S, Biancolella M, Wang TT, Treacy DJ, Luo S, Schroth GP, Housman DE, Reddy S, Lécuyer E, Burge CB: Transcriptomewide regulation of pre-mRNA splicing and mRNA localization by muscleblind proteins. Cell 2012, 150:710-724.

15. Schwartz S, Meshorer E, Ast G: Chromatin organization marks exon-intron structure. Nat Struct Mol Biol 2009, 16:990-995.

16. Tilgner H, Nikolaou C, Althammer S, Sammeth M, Beato M, Valcárcel J, Guigó R: Nucleosome positioning as a determinant of exon recognition. Nat Struct Mol Biol 2009, 16:996-1001.

17. Harper SJ, Bates DO: VEGF-A splicing: the key to anti-angiogenic therapeutics? Nat Rev Cancer 2008, 8:880-887.

18. Kalsotra A, Xiao X, Ward AJ, Castle JC, Johnson JM, Burge CB, Cooper TA: A postnatal switch of CELF and $M B N L$ proteins reprograms alternative splicing in the developing heart. Proc Natl Acad Sci USA 2008, 105:20333-20338.

19. Nowak DG, Amin EM, Rennel ES, Hoareau-Aveilla C, Gammons M, Damodoran G, Hagiwara M, Harper SJ, Woolard J, Ladomery MR, Bates DO: Regulation of Vascular Endothelial Growth Factor (VEGF) Splicing from Pro-angiogenic to Anti-angiogenic Isoforms: a Novel Therapeutic Strategy for Angiogenesis. J Biol Chem 2009, 285:5532-5540.

20. Hall MP, Nagel RJ, Fagg WS, Shiue L, Cline MS, Perriman RJ, Donohue JP, Ares M: Quaking and PTB control overlapping splicing regulatory networks during muscle cell differentiation. RNA 2013, 19:627-638.

21. Ule J, Stefani G, Mele A, Ruggiu M, Wang X, Taneri B, Gaasterland T, Blencowe BJ, Darnell RB: An RNA map predicting Nova-dependent splicing regulation. Nature 2006, 444:580-586.

22. Kawamoto S: Neuron-specific alternative splicing of nonmuscle myosin II heavy chain-B pre-mRNA requires a cis-acting intron sequence. $J$ Biol Chem 1996, 271:17613-17616.

23. Aznarez I, Barash Y, Shai O, He D, Zielenski J, Tsui LC, Parkinson J, Frey BJ, Rommens JM, Blencowe BJ: A systematic analysis of intronic sequences downstream of $5^{\prime}$ splice sites reveals a widespread role for U-rich motifs and TIA1/TIAL1 proteins in alternative splicing regulation. Genome Res 2008, 18:1247-1258

24. Chen M, Manley JL: Mechanisms of alternative splicing regulation: insights from molecular and genomics approaches. Nat Rev Mol Cell Biol 2009, 10:741-754.

25. Melamed Z, Levy A, Ashwal-Fluss R, Lev-Maor G, Mekahel K, Atias N, Gilad S, Sharan R, Levy C, Kadener S, Ast G: Alternative Splicing Regulates Biogenesis of miRNAs Located across Exon-Intron Junctions. Mol Cell 2013, 50:869-881.
26. Pruitt KD, Tatusova T, Brown GR, Maglott DR: NCBI Reference Sequences (RefSeq): current status, new features and genome annotation policy. Nucleic Acids Res 2012, 40:D130-D135.

27. Dreszer TR, Karolchik D, Zweig AS, Hinrichs AS, Raney BJ, Kuhn RM, Meyer LR, Wong M, Sloan CA, Rosenbloom KR: The UCSC Genome Browser database: extensions and updates 2011. Nucleic Acids Res 2012, 40:D918-D923.

28. Ben-Dor A, Friedman N, Yakhini Z: Scoring Genes for Relevance. Agilent; 2000.

doi:10.1186/gb-2013-14-10-r114

Cite this article as: Barash et al:: AVISPA: a web tool for the prediction and analysis of alternative splicing. Genome Biology 2013 14:R114.

\section{Submit your next manuscript to BioMed Central and take full advantage of:}

- Convenient online submission

- Thorough peer review

- No space constraints or color figure charges

- Immediate publication on acceptance

- Inclusion in PubMed, CAS, Scopus and Google Scholar

- Research which is freely available for redistribution

Submit your manuscript at www.biomedcentral.com/submit
C Biomed Central 